\title{
Determination of the mid-IR femtosecond surface-damage threshold of germanium
}

\author{
Rohit Malik ${ }^{1}$, Ben Mills ${ }^{1}$, Jonathan H. V. Price ${ }^{1, *}$, Marco Petrovich ${ }^{1}$, Zakaria \\ Moktadir $^{2}$, Zhihong $\mathrm{Li}^{1}$, Harvey N. Rutt ${ }^{1,2}$ \\ ${ }^{1}$ Optoelectronics Research Centre, University of Southampton, SO17 1BJ, UK \\ ${ }^{2}$ Department of Electronics and Computer Science, University of Southampton, \\ SO17 1BJ, UK
}

Tel. $\quad+44(0) 2380594761$

Fax. $\quad+44(0) 2380593142$

e-mail*jhvp@orc.soton.ac.uk

\begin{abstract}
To assess the potential uses of germanium as a nonlinear material in the mid-IR we have measured the surface damage threshold of germanium optical windows using femtosecond pulses at a wavelength of $3.9 \mu \mathrm{m}$. By working with a wavelength corresponding to a photon energy of less than half the band-gap energy, free carrier generation due to one and two-photon absorption was eliminated. The laser pulses had an energy of $5.5 \mu \mathrm{J}$, a duration of $255 \mathrm{fs}$ and were focused to a waist size of approximately $100 \mu \mathrm{m}$. The multi-shot damage threshold of the $\mathrm{Ge}$ windows was estimated to be approximately $94 \mathrm{GW} / \mathrm{cm}^{2}$. The data should be of immediate benefit to industrial laser researchers and applications engineers working in the mid-IR region.
\end{abstract}

Keywords: Laser damage, semiconductor, nonlinear optics, surfaces.

Abbreviations: Fourier transform infrared spectroscopy (FTIR spectroscopy); optical coherence tomography (OCT); germanium (Ge); difference frequency generation (DFG); optical parametric amplifier (OPA); second harmonic generation (SHG); full-width at half-maximum (FWHM); scanning electron micrograph (SEM).

\section{Introduction}

The mid-infrared (mid-IR) wavelength region (3-10 $\mu \mathrm{m})$ is of paramount importance to a host of industrially and scientifically relevant applications in sensing, spectroscopy and imaging. Examples include Fourier transform infrared (FTIR) spectroscopy, range finding, hyperspectral imaging, medical imaging with optical coherence tomography (OCT), chemical sensing, and pollution monitoring based on molecular spectroscopy. However, the existing sources are either of low brightness (thermal sources), highly complex and costly, or operate at a restricted set of wavelengths. Despite impressive improvements in recent years, quantum cascade lasers have narrow linewidth which makes them unsuitable for applications requiring broadband excitation. Fiber-based supercontinuum sources are already widely used in the visible and near-IR and they show great promise for the mid-IR region. However, above $2 \mu \mathrm{m}$, the high intrinsic absorption of silica means that infrared-transmitting non-silica fibers are needed for mid-IR continuum generation ${ }^{1-4}$. Crystalline materials, on the other hand, have been used for many years to 
manufacture high-quality IR optical elements such as windows and lenses, and there has been renewed interest recently in using $\mathrm{Ge}$ and $\mathrm{Si}$ as nonlinear materials (see Ref. ${ }^{5}$ and references therein for work with Silicon). Germanium is an excellent candidate for nonlinear applications in the mid-IR due to its wide transmission window $(1.8-11 \mu \mathrm{m})$ and high third order optical nonlinearity ${ }^{6}\left(\chi^{(3)}{ }_{1111}=19.2 \cdot 10^{-12} \mathrm{~m}^{2} / \mathrm{V}^{2}\right.$ at $\left.3.39 \mu \mathrm{m}\right){ }^{7}$. These properties make it particularly suitable as a nonlinear material when paired with input pulses from practical fiber laser pumped optical parametric oscillators (OPOs) ${ }^{8,9}$.

When assessing the suitability of a material, the damage threshold at the pump wavelength is often of crucial importance as nonlinear processes typically use short pulses to achieve the requisite peak power. In applications, a component would typically be irradiated with a pulse-train over an extended period so the relevant damage threshold is the multi-shot value not the singlepulse value. The laser wavelength may also have an influence on the damage threshold and working above $\approx 3.7 \mu \mathrm{m}$ is attractive because single or two-photon-absorption (TPA) related carrier generation cannot occur and thus free carrier generation is minimized.

Only very limited data exist in the literature for Ge, and for femtosecond (fs) seed pulses there is no information on the damage threshold at mid-IR wavelengths. An introduction to earlier studies with longer pulses or at shorter wavelengths can be found in the references for both crystalline ${ }^{10-22}$ and amorphous $\mathrm{Ge}^{23}$. (All of the previous picosecond and femtosecond damage studies were performed with visible or near-IR wavelengths so the photon energies were above band-gap.) In this paper we have used a laser system operating at a wavelength of $3.9 \mu \mathrm{m}$ to perform surface damage threshold measurements for multiple Ge samples scanned through the focus of the femtosecond pulse-train. The samples were commercially available Ge optical windows from Crystran (www.crystran.co.uk), obtained from an n-type (antimony doped) Ge ingot. The windows were $2 \mathrm{~mm}$ thick, diamond polished on both surfaces and cut with (111) face side. The resistivity and the carrier concentration of the Ge sample were determined using Hall measurements. The measured resistivity was $1.74 \mathrm{ohm} . \mathrm{cm}$, while the carrier concentration was $1.6 \times 10^{15} \mathrm{~cm}^{-3}$.

The paper first describes the experimental setup used to characterize the laser source and to position the sample. Next, the beam characteristics are presented and the results of damage to the sample surface are shown. A brief discussion then follows together with a conclusion.

\section{Experimental set-up}

The experimental setup is shown in Fig. 1. Femtosecond pulses at a wavelength of $3.9 \mu \mathrm{m}$ were generated using difference frequency generation (DFG) from the signal and idler of an optical parametric amplifier (OPA, Coherent OPerA) pumped by a Coherent Mira/Legend laser system. The $3.9 \mu \mathrm{m}$ wavelength was chosen because it provided the highest output power from the laser system at a wavelength above the TPA threshold for Ge. The $3.9 \mu \mathrm{m}$ source had an average power of $5.5 \mathrm{~mW}$, repetition rate of $1 \mathrm{kHz}$ and pulse duration of $255 \mathrm{fs}$. The residual signal and idler after the DFG crystal were blocked using a long-wave-pass filter (Spectrogon LP-2500nm). We confirmed the effectiveness of the filter by blocking either the signal or the idler to prevent the DFG process, and hence leave just the unblocked idler or signal, and that with no DFG, the power transmitted through the filter dropped to below the sensitivity level of our power meter.

The laser output was collimated using a telescope (L1=100 $\mathrm{mm}$ and $\mathrm{L} 2=150 \mathrm{~mm}$ ). For alignment, a co-propagating red HeNe laser was introduced via a removeable mirror (M2). A $\mathrm{f}=75 \mathrm{~mm}$ focusing lens (L3) created a waist that was used for both beam characterisation purposes and for the damage measurements. All lenses were Calcium Fluoride $\left(\mathrm{CaF}_{2}\right)$, anti-reflection coated at $3.9 \mu \mathrm{m}$ and had plano-convex form. Neutral density filters were used to attenuate the beam in order to avoid damage to the detector while performing knife-edge scans across the beam ${ }^{24}$. An additional lens (L4) was used to reduce the beam size to completely fit onto a liquid Nitrogen cooled InSb detector. As shown in section 3, the knife-edge data points were fitted to a Gaussian beam profile in order to obtain the $\mathrm{M}^{2}$ values for the horizontal and vertical axes ${ }^{24}$. 

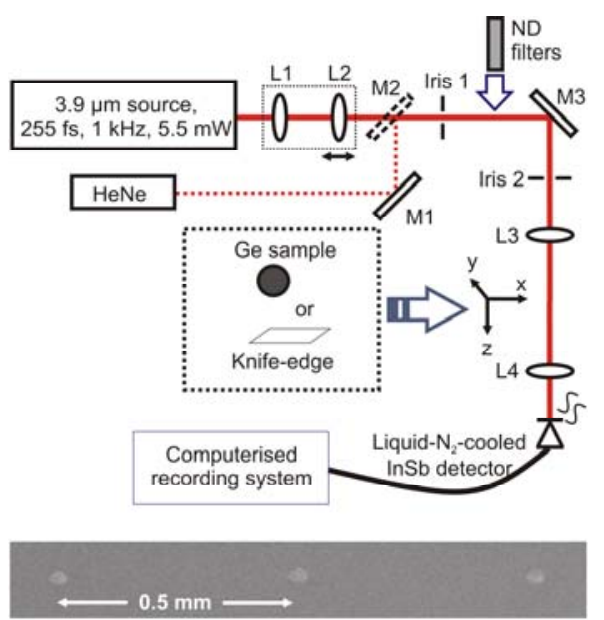

Fig.1. Experimental set-up for the beam characterization and for positioning the Ge sample.

Inset: SEM image showing the sequence of damage spots used to confirm the reproducibility of results. (Enlarged SEM images are shown in Fig. 3)

For the damage experiment, the knife edge was replaced by a Ge sample. To change the intensity, we chose to vary the size of the focus instead of attenuating the beam (e.g. by the use of filters) which could have altered the duration of the pulses. Placing the Ge sample precisely at the focus was challenging because there was no unambiguous, real-time, indication of the waist position (i.e. the position of the circle of least confusion between the horizontal and vertical foci). At wavelengths close to $800 \mathrm{~nm}$, other authors have reported using surface second harmonic generation (SHG) to directly detect when the sample was at the position of the beam-waist ${ }^{18,25}$. In addition, the low pulse energies (compared to those available closer to the $800 \mathrm{~nm}$ fundamental wavelength from the Ti:Sapphire laser) led to a requirement for a small waist size and this produced a short Rayleigh length over which the maximum intensity could be maintained. Therefore, we used a scanning technique similar to that used by Joglekar et al. ${ }^{26}$. Then, the sample was scanned through the waist at a rate of $0.1 \mathrm{~mm} / \mathrm{s}$. The scans were started 2 or 3 Rayleigh ranges behind the waist position and finished a similar distance in front of the waist position to ensure the foci in horizontal and vertical directions were included. A typical scan required $\sim 40-60$ seconds to traverse the Rayleigh distance. The beam was then blocked whilst translating the sample back to the original axial position and moving it sideways. The scan was repeated to create sets of damage spots separated by a known distance.

The motorized stage was aligned parallel with the beam, and for each focal spot size, a knifeedge was used to measure the waist of the beam incident on the focusing lens. The waist size was changed by adjusting the distance between the telescope lenses (L1, L2) in order to vary the beam size on the focusing lens (L3).

As a precaution, we always scanned the Ge sample towards the laser so that the front surface would pass through the focus before the back surface since both self-focusing and enhancement of the local intensity due to superposition of incident and reflected pulses can increase the intensity at the back surface of the sample ${ }^{25}$. However, in practice no damage was observed at the rear facet of the sample, which was probably due to the high Fresnel loss $(\sim 36 \%$ at $3.9 \mu \mathrm{m})$ at the front surface. As we were measuring the front surface damage threshold the numerical-aperture (NA) of the incident beam was not as critical as in bulk damage experiments where the NA affects the length scale over which self-focusing can occur.

\section{Beam characterization results}

The beam characterization data and the fitted $\mathrm{M}^{2}$ curves are shown in Fig. 2(a). We attribute the astigmatism to the geometry used for the nonlinear generation process in the OPA and DFG systems. The focal spot size was calculated by considering the beam as an ellipse with Gaussian intensity profiles in horizontal and vertical directions. Using the Gaussian beam propagation equations to perform a nonlinear least squares fit to the data gave $\mathrm{M}^{2}$ values in horizontal and vertical directions of 2.0 and 2.35 respectively. We measured the beam width on the focusing lens, the measured distances between the lenses and the $\mathrm{M}^{2}$ parameters in the horizontal and vertical directions and then standard ABCD ray transfer matrix calculations were used to estimate the 
radius of curvature of the beam at the focusing lens (L3) ${ }^{27}$. Initially the measurements were performed for the beam-size that was closest to the damage threshold $\left(94 \mathrm{GW} / \mathrm{cm}^{2}\right)$ and the minimum horizontal and vertical widths were $326 \mu \mathrm{m}$ and $188 \mu \mathrm{m}$, respectively.

The spectrum shown in Fig. 2(b) was measured using a Bentham Monochromator (TMc300) and cooled InSb detector. The full width half maximum (FWHM) was $100 \mathrm{~nm}$ and the profile of the spectrum was Gaussian.

The pulse duration was measured using a home-built noncollinear autocorrelator with an extended indium gallium arsenide ( $\mathrm{InGaAs}$ ) diode as a TPA detector ${ }^{28}$. One arm of the autocorrelator incorporated a computer-controlled motorized stage to vary the delay. The beam path for the autocorrelation contained all of the optical elements used when focusing the beam onto the sample so the pulse broadening due to dispersion of the lenses was included in the measured duration. The autocorrelation trace with background removed is shown inset to Fig. 2(b). Fitting to a Gaussian profile indicated an autocorrelation duration (FWHM) of $362 \mathrm{fs}$ and an equivalent Gaussian pulse duration of $255 \mathrm{fs}$ (FWHM). The flat-phase Fourier transform of the spectrum indicated a pulse duration of $220 \mathrm{fs}$ (FWHM), so the measured pulses were close to transform limited.
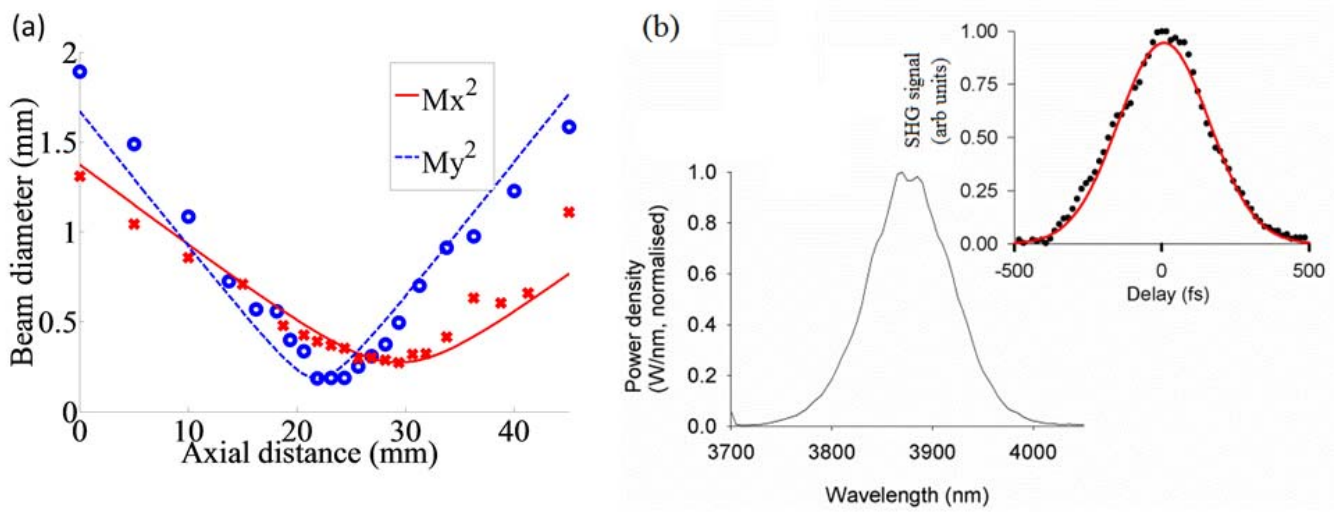

Fig.2. (a) Fitted $\mathrm{M}^{2}$ curves in the horizontal-direction (red solid line) and in the vertical-direction (dotted blue line). (b) Spectrum of the $3.9 \mu \mathrm{m}$ beam with autocorrelation shown inset.

The average pulse energy was calculated by dividing the average power by the pulse repetition rate. We also used a cooled InSb detector connected to an oscilloscope (Agilent MSO7104) to measure the pulse-to-pulse fluctuations. The beam was attenuated using neutral density filters to avoid damage to the detector and the beam diameter was reduced using a lens to ensure that it completely fitted onto the detector. The statistical fluctuations of the pulse amplitude were recorded over an interval of 15 seconds which was representative of the time required to scan the sample through the focus. The absolute max/min peak positions were $+/-3 \%$ either side of the mean peak position and the standard deviation was $+/-1.2 \%$ of the mean peak position. The variation was probably due to air currents in the laboratory leading to small beam deflections affecting the efficiency of the nonlinear frequency down-conversion processes.

\section{Damage threshold measurement results}

The criterion for damage was observation of changes on the sample when viewed using an optical microscope with 500 times magnification i.e. $50 \mathrm{x}$ objective lens; $10 \mathrm{x}$ eyepiece lens. (The use of a Normarski phase contrast microscope, as typically employed to characterize structural changes to transparent dielectrics is not suitable for Ge as it is not transparent in the visible region.) We found that the lowest intensity for which damage was observable on the sample surface was $94 \mathrm{GW} / \mathrm{cm}^{2}$ and the highest intensity for which no damage was observable was $74 \mathrm{GW} / \mathrm{cm}^{2}$. Therefore the structural damage threshold was within this range. For each intensity value, three areas on the sample were tested which confirmed the reproducibility of the data. Measurements were repeated on a second Ge sample and identical damage threshold results were obtained. This indicates that the damage threshold was unlikely to have been an artifact of either a particular sample or region on a sample. The damage threshold value of $94 \mathrm{GW} / \mathrm{cm}^{2}$ corresponds to a fluence of $0.026 \mathrm{~J} / \mathrm{cm}^{2}$.

Images from a scanning electron microscope (SEM) are shown in Fig. 3. Damaged regions created slightly above damage threshold $\left(94 \mathrm{GW} / \mathrm{cm}^{2}\right)$ are shown in (a)-(c), and at increasing intensities above threshold of $103 \mathrm{GW} / \mathrm{cm}^{2}$ in (d)-(f) and $155 \mathrm{GW} / \mathrm{cm}^{2}$ in (g)-(i). It is clear that as 
the intensity is increased, the damaged region becomes larger and that, at the highest intensity the indentation is also deeper. While (a)-(c) show relatively round patterns, (d)-(f) show greatly elongated or even double spot damage and (g)-(i) also show elongation. Hence, the spatial energy distribution was rather irregular as would be expected for the astigmatic beam.

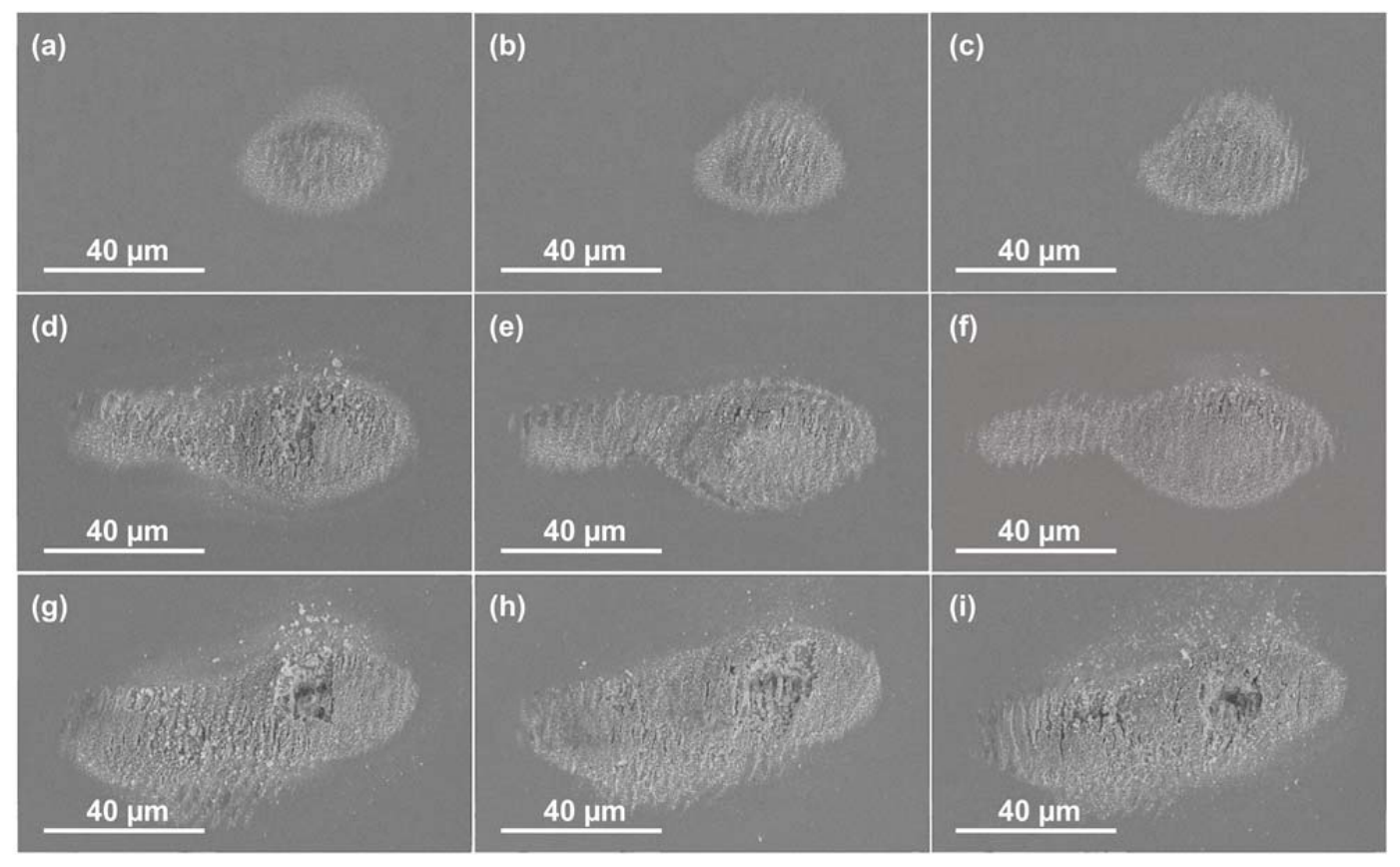

Fig.3. SEM images of damage spots as a result of irradiation with the $3.9 \mu \mathrm{m}$ beam, for intensities of (a)-(c) $94 \mathrm{GW} / \mathrm{cm}^{2}$, (d)-(f) $103 \mathrm{GW} / \mathrm{cm}^{2}$, and (g)-(i) $155 \mathrm{GW} / \mathrm{cm}^{2}$. The laser polarization was horizontal with respect to the images. The damage threshold measurement was repeated three times for each intensity.

Whilst the damage features observed in the femtosecond regime are generally highly reproducible from shot-to-shot ${ }^{29}$, there are small differences between the SEM images in Fig. 3. We attribute the variability to mirror vibrations during the scans. Another feature visible on the SEM images in Fig. 3 is the ripple pattern. Ripples have been shown to build up gradually after successive pulses with energy above the damage threshold are incident on a surface and are quite commonly observed ${ }^{30,31}$. Sipe et al. studied ripple formation with much longer pulses at a shorter wavelength and suggested that at normal incidence the ripple period should equal the wavelength and should be perpendicular to the polarization ${ }^{32,33}$. However, it appears that with femtosecond pulses this simple relationship between wavelength and ripple spacing is no longer valid and subwavelength ripples are often observed ${ }^{34-37}$. Recent experimental and modelling work has been directed towards obtaining an understanding of periodic structure formation process and controlling the ripple profile. This is an area of technological importance for a wide range of applications requiring precise surface structuring. The research has identified that the ripple parameters depend on both the number of incident pulses and on the fluence and pulse duration and provided detailed numerical models that provide agreement with the structures observed experimentally. Figure 3 shows that in our experiment the ripple spacing was $3.2 \mu \mathrm{m}$, which is indeed sub-wavelength, and that the ripples are close to (although not precisely) perpendicular to the horizontal polarization of the incident light.

\section{Analysis and discussion}

At room temperature, intrinsic Ge has an indirect-bandgap transition-energy of $0.66 \mathrm{eV}$. Hence photons with wavelengths below $1.85 \mu \mathrm{m}$ have sufficient energy to promote carriers from valence to conduction bands. As Ge is an indirect bandgap material, the absorption coefficient increases dramatically for photons with a wavelength below $1.55 \mu \mathrm{m}$ as they have sufficient energy to excite 
carriers at band centre. (The direct bandgap energy is $0.8 \mathrm{eV}$.) At wavelengths between $1.85 \mu \mathrm{m}$ and $3.7 \mu \mathrm{m}$, carrier creation requires at least two-photons and so the absorption coefficient drops by orders of magnitude compared to the value at shorter wavelengths. For even longer wavelengths, three or more photons are needed to create mobile carriers.

Although Ge is a widely used optical material in the mid-IR, it is notable that the material normally used is lightly-doped n-type Ge, not intrinsic Ge, due to a subtlety of the valence band structure. In p-type and intrinsic Ge, transitions of holes between valence sub-bands lead to three strong IR absorption bands in the $2-10 \mu \mathrm{m}$ region ${ }^{38-40}$. There is also a low density of thermally excited electrons that leads to a background Drude-Zener type absorption with an approximately $\lambda^{2}$ dependence. For optical components, an optimum level of n-type doping is therefore chosen to balance the suppression of the intra-valence-band transitions of holes and the increase in DrudeZener absorption due to the excess electrons ${ }^{41,42}$. Thus at $3.9 \mu \mathrm{m}$ in typical $1-10$ ohm.cm optical grade germanium the dominant residual linear absorption mechanisms are weak DrudeZener absorption by both electrons and holes and weak spin-orbit split off valence band to heavy hole absorption by holes.

At $3.9 \mu \mathrm{m}$, where one and two-photon carrier excitation are forbidden, there have been no damage studies investigating Ge optics with femtosecond sources. However, there are a wide range of studies investigating the damage mechanisms of wide-gap transparent dielectrics which provide useful background to the processes likely to occur with Ge optics. (These earlier studies were performed due to the availability of femtosecond laser sources and the relevance of the materials for laser optics and materials processing ${ }^{26,29,41-48}$.) Theoretical and experimental results indicate that for pulses with durations above $\sim 1-10 \mathrm{ps}$, the damage mechanism in wide-gap dielectrics is thermal melting and hence the features which are created show some variability. For femtosecond pulses, the initial free electron generation may be followed by non-thermal plasma creation ${ }^{29}$ or nonthermal melting and the formation of an overcritical fluid leading via nucleation to a rapid transition to vapour and liquid droplets that can be ejected ${ }^{26,29,35}$

We note that the critical plasma density for damage in wide-gap dielectrics is typically $\sim 1.6 \times 10^{21} \mathrm{~cm}^{-3}$ for femtosecond pulses at a laser wavelength of $800 \mathrm{~nm}$ because the material becomes highly absorbing when the free electron density exceeds the plasma density ${ }^{47}$. At $3.9 \mu \mathrm{m}$ the critical plasma density reduces to $\sim 7 \times 10^{19} \mathrm{~cm}^{-3}$. The room temperature carrier concentrations of intrinsic and n-type "optical" Ge of $\sim 3 \times 10^{13} \mathrm{~cm}^{-3}$ and $1.6 \times 10^{15} \mathrm{~cm}^{-3}$ respectively are at least $10^{4}$ below that required for ablation. Brief calculations for three photon absorption carrier generation using parameters measured at a similar wavelength by Seo et al. ${ }^{49}$ and based on their equation (3) suggest that for a single pulse with fluence of $26 \mathrm{~mJ} / \mathrm{cm}^{2}$ on a Ge surface the carrier density would be well below that required for melting to occur. (We have used energy balance to calculate the temperature change that would occur on carrier recombination.) Therefore accumulated defects from a train of pulses are an important modifying factor ${ }^{50}$. Studies with ps pulses on Ge at a wavelength of $584 \mathrm{~nm}^{51}$ are not directly comparable because at that wavelength, single photon carrier generation is possible. Studies with longer pulses ${ }^{52}$ may involve processes that occur within the duration of the pulse (e.g. carrier recombination and lattice heating), whereas for fs pulses, thermalisation of the carriers created occurs on timescales longer than the excitation pulse. Similar caution is applicable when comparing the results here with previous work at shorter wavelengths showing that if sufficient free-carrier absorption occurred on the (non-thermal) timescale over which the laser pulse deposits energy into the sample and then once $>10 \%$ of carriers from the valence band were non-thermally excited the (cold) lattice weakens and structural changes readily occur ${ }^{44,45}$. Additional data would be necessary in order to determine the precise processes involved in this experiment.

The damage threshold can be altered by variations in the surface roughness and because of the so-called "incubation effect", whereby the damage threshold decreases below the single-shot damage intensity as a sample is irradiated with a succession of sub-threshold pulses. Previous studies ${ }^{53}$, the symposium series on Laser-Induced Damage in Optical-Materials ${ }^{15,54}$ and reports from the symposia in subsequent years, refer to evidence that increased roughness leads to a reduction in the damage threshold ${ }^{25,50}$. In addition, the incubation effect ${ }^{46,55}$ can reduce the damage threshold by up to $70 \%$, compared to the single-shot value, due to the formation of defects 46

In this work, the sample was scanned through the focus at a rate of $0.1 \mathrm{~mm} / \mathrm{s}$ and a typical scan through the focus required $\sim 40-60$ seconds to complete which, with the laser repetition rate of $1 \mathrm{kHz}$, resulted in $\sim 10,000$ pulses having been incident on the sample before the waist position was reached. Since it has shown that the incubation effect typically saturates after 100 to 1000 pulses ${ }^{46}$, the incubation effect is believed to have saturated in our experiment. The first row of 
Table 1 shows the number of pulses incident on the Ge crystal within the confocal parameter (i.e. 2 $x$ Rayleigh range) of the focus at the intensity that marked the onset of damage. This corresponds to the scan used to process the sample shown in the SEM images in Fig. 3 (a)-(c).

Table 1 also shows the number of pulses calculated to be within the confocal parameter for the intensities used to create the damage features shown in Fig. 3 (d)-(f) and (g)-(i). For the higher intensity damage there was a region of the scan below the damage intensity providing the incubation effect, and then the onset of damage would occur. Further translation through the focus would then create additional damage until the scan went back to a region where the intensity was below the damage threshold. To provide an indication of how a variable intensity profile was applied to the sample in this experiment the calculated pulse numbers in the three regions is analysed in Table 1 (and the on-axis intensity is presented in Fig. 4).

\begin{tabular}{|c|c|c|c|c|}
\hline \multirow{2}{*}{$\begin{array}{r}\text { Peak } \\
\text { Intensity } \\
(\mathrm{GW} / \\
\left.\mathrm{cm}^{2}\right)\end{array}$} & \multirow{2}{*}{$\begin{array}{r}\text { Rayleigh } \\
\text { Range }\left(\mathrm{z}_{\mathrm{R}}\right)\end{array}$} & \multicolumn{3}{|c|}{ Number of laser pulses above and below $\mathrm{I}_{\mathrm{TH}}$} \\
\hline & & $\begin{array}{r}\text { Incubation region } \\
\qquad\left(\mathrm{I}<\mathrm{I}_{\mathrm{TH})}\right)\end{array}$ & $\begin{array}{r}\text { Material } \\
\text { modification } \\
\text { region }\left(\mathrm{I}>\mathrm{I}_{\mathrm{TH}}\right)\end{array}$ & $\begin{array}{r}\text { After } \\
\text { modification } \\
\text { region }\left(\mathrm{I}<\mathrm{I}_{\mathrm{TH}}\right)\end{array}$ \\
\hline 94 & 6.2 & $\begin{array}{r}61,500 \\
(50 \%)\end{array}$ & NA & $\begin{array}{r}61,500 \\
(50 \%)\end{array}$ \\
\hline 103 & 5.6 & $\begin{array}{r}39,200 \\
(35 \%)\end{array}$ & $\begin{array}{r}33,800 \\
(30 \%)\end{array}$ & $\begin{array}{r}39,200 \\
(35 \%)\end{array}$ \\
\hline 155 & 3.7 & $\begin{array}{r}7,300 \\
(10 \%)\end{array}$ & $\begin{array}{r}60,100 \\
(81 \%)\end{array}$ & $\begin{array}{r}7,300 \\
(10 \%)\end{array}$ \\
\hline
\end{tabular}

Table.1. Number of pulses below and above the threshold intensity $\left(\mathrm{I}_{\mathrm{TH}}\right)$ as the sample was scanned through the confocal parameter for the three sets of damage images shown in Fig. 3. .

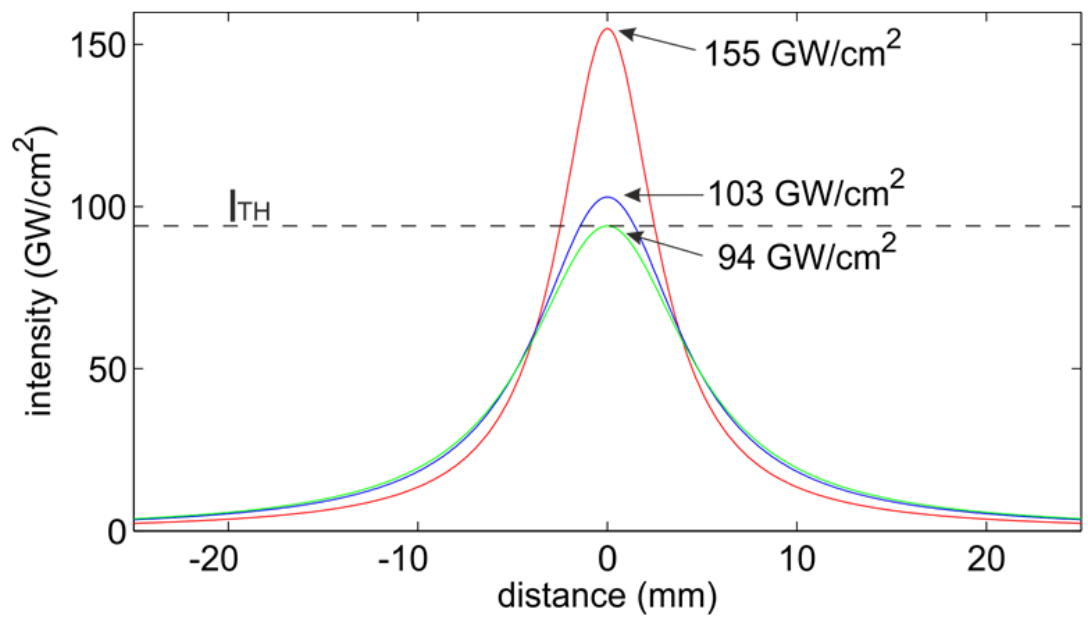

Fig.4. On-axis intensity of the beam, at positions through the focus, for the three sets of damage images shown in Fig. 3.

We expect that improvements to the techniques used here may be implemented in the future, but note that mid-IR studies are currently challenging to perform as they require building the beam characterization equipment in-house and because beam viewers or fluorescent cards are not readily available. Against this background, our data is the first to use femtosecond pulses at a wavelength where more than two photons are required for carrier creation. The combination of n-type substrate, multi-shot data and weakly multimode beam corresponds to the setup typically encountered in applications based environments and represents a practical lower limit on the damage threshold appropriate for field-based systems. The measurement should be of immediate practical benefit to industrial scientists and laser engineers. 


\section{Conclusion}

Nonlinear optics in the mid-IR wavelength region is a rapidly evolving research field with the potential for developing a new generation of practical, high-brightness broad-bandwidth sources. Particularly attractive mid-IR sources include the emerging technology of rare-earth doped fiber pumped OPO/OPA systems. The addition of a crystal based element for broadening the wavelength-span is an exciting possibility as it would enable the creation of broad bandwidth sources for a range of novel applications. Germanium is both a well-known infrared optical material and an excellent candidate for nonlinear applications in the mid-IR region due to its wide transmission window $(1.8-11 \mu \mathrm{m})$ and high third order optical nonlinearity. However, until now, ultra-short pulse damage threshold measurements on Ge have not been measured at mid-IR pump wavelengths.

We report the first measurement of the damage threshold of polished Ge using $255 \mathrm{fs}$ pulses at a laser wavelength of $3.9 \mu \mathrm{m}$ i.e. at a wavelength where one and two-photon-absorption induced free carrier creation are prohibited. The sample was a lightly $\mathrm{n}$-doped commercially available diamond polished Ge optical window. The damage threshold was found to be approximately $94 \mathrm{GW} / \mathrm{cm}^{2}$. The data should be of immediate use to industrial and applicationsbased researchers requiring field-based reliability data on Ge optics for a wide-range of novel future sources.

\section{Acknowledgements:}

We thank Professor R.W. Eason, Dr. C. Corbari, Dr. P. Horak, Dr. J. C. Gates, Dr. A.J. Heidt, Dr. N. Bulgarkova, Mr. T. Franklin, Mr. F. Kienle and Professor D. J. Richardson for valuable discussions or assistance with the measuring equipment used. This work was supported in part by the EPSRC (UK) grant EP/H049371/1. Dr. J. Price was supported by a Royal Academy of Engineering/EPSRC (UK) Research Fellowship. 


\section{References:}

1 J. H. Price, T. M. Monro, H. Ebendorff-Heidepriem, F. Poletti, P. Horak, V. Finazzi, J. Y. Y. Leong, P. Petropoulos, J. C. Flanagan, G. Brambilla, $\mathrm{X}$. Feng, and D. J. Richardson, IEEE Journal of Selected Topics in Quantum Electronics 3, 738 (2007).

J. H. V. Price and D. J. Richardson, in Supercontinuum Generation in Optical Fibers, edited by J. M. Dudley and R. Taylor (Cambridge University Press (CUP), Cambridge, 2010), p. 82.

C. A. Xia, Z. Xu, M. N. Islam, F. L. Terry (Jr.), M. J. Freeman, A. Zakel, and J. Mauricio, IEEE Journal Of Selected Topics In Quantum Electronics 15, 422 (2009).

P. Domachuk, N. A. Wolchover, M. Cronin-Golomb, A. Wang, A. K. George, C. M. B. Cordeiro, J. C. Knight, and F. G. Omenetto, Opt Express 16,7161 (2008).

B. Jalali, Nature Photonics 4, 506 (2010).

N. K. Hon, R. Soref, and B. Jalali, Journal of Applied Physics 110, 011301 (2011).

N. P. Barnes and M. S. Piltch, J. Opt. Soc. Am. 69, 178 (1979).

S. Hädrich, J. Rothhardt, F. Röser, T. Gottschall, J. Limpert, and A. Tünnermann, Optics Express 16, 19812 (2008).

M. V. O'Connor, M. A. Watson, D. P. Shepherd, D. C. Hanna, J. H. V. Price, A. Malinowski, J. Nilsson, N. G. R. Broderick, and D. J. Richardson, Optics Letters 27, 1052 (2002).

J. R. Meyer, M. R. Kruer, and F. J. Bartoli, Journal of Applied Physics 51, 5513 (1980).

R. M. Wood, GEC-Journal of Science \& Technology 45, 109 (1979).

R. S. Taylor, B. K. Garside, and E. A. Ballik, IEEE Journal of Quantum Electronics 14, 532 (1978).

M. Bass and K. M. Leung, IEEE Journal of Quantum Electronics 12, 82 (1976).

L. J. Willis and D. C. Emmony, Optics and Laser Technology 7, 222 (1975).

A. J. Glass and A. H. Guenther, Applied Optics 15, 1510 (1976).

D. H. Auston and C. V. Shank, Physical Review Letters 32, 1120 (1974). 
A. Elci, M. O. Scully, A. L. Smirl, and J. C. Matter, Physical Review B 16, 191 (1977).

M. I. Park, C. O. Park, C. S. Kim, and S. C. Jeoung, Journal of the Korean Physical Society 46, 531 (2005).

A. P. Singh, A. Kapoor, and K. N. Tripathi, Optics and Laser Technology 35, 659 (2003).

A. P. Singh, A. Kapoor, and K. N. Tripathi, Optics and Laser Technology 35, 87 (2003).

A. Cavalleri, C. W. Siders, C. Rose-Petruck, R. Jimenez, C. Toth, J. A. Squier, C. P. J. Barty, K. R. Wilson, K. Sokolowski-Tinten, M. H. von Hoegen, and D. von der Linde, Physical Review B 63, 3306 (art. no. 193306) (2001).

C. W. Siders, A. Cavalleri, K. Sokolowski-Tinten, C. Toth, T. Guo, M. Kammler, M. H. von Hoegen, K. R. Wilson, D. von der Linde, and C. P. J. Barty, Science 286, 1340 (1999).

O. Salihoglu, U. Kurum, H. G. Yaglioglu, A. Elmali, and A. Aydinli, Journal of Applied Physics 109 (2011).

A. E. Siegman, M. W. Sasnett, and T. F. Johnston, IEEE Journal of Quantum Electronics 27, 1098 (1991).

A. V. Smith and B. T. Do, Applied Optics 47, 4812 (2008).

A. P. Joglekar, H. Liu, G. J. Spooner, E. Meyhofer, G. Mourou, and A. J. Hunt, Applied Physics B-Lasers and Optics 77, 25 (2003).

B. Mills, E. T. F. Rogers, J. Grant-Jacob, S. L. Stebbings, M. Praeger, A. M. de Paula, C. A. Froud, R. T. Chapman, T. J. Butcher, W. S.

Brocklesby, and J. G. Frey, Proceedings of the SPIE - The International Society for Optical Engineering 7360 (2009).

Y. Takagi, T. Kobayashi, K. Yoshihara, and S. Imamura, Optics Letters 17, 658 (1992).

S. S. Mao, F. Quere, S. Guizard, X. Mao, R. E. Russo, G. Petite, and P. Martin, Applied Physics A-Materials Science \& Processing 79, 1695 (2004).

C. F. Kaminski, R. S. Watt, A. D. Elder, J. H. Frank, and J. Hult, Applied Physics B-Lasers and Optics 92, 367 (2008).

J. Reif, O. Varlamova, M. Ratzke, M. Schade, H. S. Leipner, and T. Arguirov, Applied Physics A-Materials Science \& Processing 101, 361 (2010).

J. E. Sipe, J. Young, J. S. Preston, and H. M. Driel, Phys. Rev. B 27, 1141 (1983). 

(1983).

G. D. Tsibidis, M. Barberoglou, P. A. Loukakos, E. Stratakis, and C. Fotakis, Physical Review B 86 (2012).

F. Garrelie, J. P. Colombier, F. Pigeon, S. Tonchev, N. Faure, M.

Bounhalli, S. Reynaud, and O. Parriaux, Optics Express 19, 9035 (2011).

J. Bonse, A. Rosenfeld, and J. Krueger, Applied Surface Science 257, 5420 (2011).

J. Bonse and J. Krueger, Journal of Applied Physics 108 (2010).

M. Woerner, T. Elsaesser, and W. Kaiser, Physical Review B 45, 8378 (1992).

W. E. Pinson and R. Bray, Physical Review 136, A1449 (1964).

W. Kaiser, R. J. Collins, and H. Y. Fan, Physical Review 91, 1380 (1953).

A. H. Kahn, Physical Review 97, 1647 (1955).

H. Y. Fan, W. Spitzer, and R. J. Collins, Physical Review 101, 566 (1956).

D. Du, X. Liu, G. Korn, J. Squier, and G. Mourou, Applied Physics Letters 64, 3071 (1994).

S. K. Sundaram and E. Mazur, Nature Materials 1, 217 (2002).

F. Rossi and T. Kuhn, Reviews of Modern Physics 74, 895 (2002).

A. Rosenfeld, M. Lorenz, R. Stoian, and D. Ashkenasi, Applied Physics A-Materials Science \& Processing 69, S373 (1999).

A. C. Tien, S. Backus, H. Kapteyn, M. Murnane, and G. Mourou, Physical Review Letters 82, 3883 (1999).

E. G. Gamaly, Physics Reports 508, 91 (2011).

D. Seo, J. M. Gregory, L. C. Feldman, N. H. Tolk, and P. I. Cohen, Physical Review B 83 (2011).

S. V. Vintsents, V. B. Zaitsev, A. V. Zoteev, G. S. Plotnikov, A. I. Rodionov, and A. V. Chervyakov, Semiconductors 36, 883 (2002).

N. Chaoui, J. Siegel, S. M. Wiggins, and J. Solis, Applied Physics Letters 86 (2005).

N. Chaoui, J. Siegel, J. Solis, and C. N. Afonso, Journal of Applied Physics 89, 3763 (2001).

A. V. Kuanr, S. K. Bansal, and G. P. Srivastava, Optics and Laser Technology 28, 345 (1996). 
A. J. Glass and A. H. Guenther, Applied Optics 16, 1214 (1977).

55 F. Costache, S. Eckert, and J. Reif, Applied Physics A-Materials Science \& Processing 92, 897 (2008). 